Pacific

Journal of

Mathematics

A REMARK ON KHOVANOV HOMOLOGY AND TWO-FOLD BRANCHED COVERS

LIAM WATSON

Volume $245 \quad$ No. 2

April 2010 


\title{
A REMARK ON KHOVANOV HOMOLOGY AND TWO-FOLD BRANCHED COVERS
}

\author{
LIAM WATSON
}

\begin{abstract}
We give examples of knots distinguished by the total rank of their Khovanov homology but sharing the same two-fold branched cover. Hence Khovanov homology does not yield an invariant of two-fold branched covers.
\end{abstract}

Mutation provides an easy method for producing distinct knots sharing a twofold branched cover: The mutation in the branch set corresponds to a trivial surgery in the cover. Due to a result of Wehrli [2007; 2009] (see also [Bloom 2009]), this provides a range of examples of manifolds that branch cover $S^{3}$ in more than one way, but for which the distinct branch sets have identical rank in their respective Khovanov homology groups over $\mathbb{F}_{2}=\mathbb{Z} / 2 \mathbb{Z}$.

From this point of view this fact is not completely surprising, as Khovanov homology is closely related to the Heegaard Floer homology of two-fold branched covers [Ozsváth and Szabó 2005]. Indeed, this is made precise in Bloom's proof of mutation invariance [2009]. More generally, there is a question posed by Ozsváth: Is Khovanov homology an invariant of the two-fold branched cover? More precisely, is the total rank of the reduced Khovanov homology (over $\mathbb{F}_{2}$ ) an invariant of two-fold branched covers? This short note gives a negative answer.

Theorem. The total rank of Khovanov homology is not an invariant of two-fold branched covers.

This theorem is proved by exhibiting manifolds that are two-fold branched covers of $S^{3}$ in two different ways, and for which the pair of branch sets is distinguished by the total rank in Khovanov homology. We work with the reduced version of Khovanov homology, denoted $\widetilde{\mathrm{Kh}}$, with $\mathbb{F}_{2}$ coefficients [Khovanov 2000; 2003].

Surgery on torus knots. Let $S_{r / s}^{3}(K)$ denote the result of $(r / s)$-surgery on a knot $K \hookrightarrow S^{3}$, and let $T_{p, q}$ denote the positive $(p, q)$ torus knot in $S^{3}$ (with $0<p<q$ ). Note that, as we will only consider torus knots, $p$ and $q$ are relatively prime.

Proposition 1 [Moser 1971]. The manifold $S_{ \pm 1 / n}^{3}\left(T_{p, q}\right)$ is Seifert fibered with base orbifold $S^{2}(p, q, p q n \mp 1)$ for $n>0$.

MSC2000: 57M12, 57M27.

Keywords: Khovanov homology, two-fold branched cover, Heegaard Floer homology.

Supported by a Canada Graduate Scholarship (NSERC). 
Our conventions for Seifert fibered spaces follow [Boyer 2002]. Our conventions differ from those in Moser's work, resulting in the sign discrepancy between our statement and Moser's. By applying the work of Heil [1974], it is possible to give a quick proof:

Proof. Let $M=S^{3} \backslash \nu\left(T_{p, q}\right)$, so that $M(\alpha)=S_{r / s}^{3}\left(T_{p, q}\right)$ for a given slope $\alpha=r \mu+s \lambda$, where $\mu$ is the knot meridian and $\lambda$ is the preferred longitude. As the complement of a regular fiber of a Seifert fibration of $S^{3}, M$ is Seifert fibered with base orbifold $D^{2}(p, q)$. Let $\varphi$ denote a regular fiber in $\partial M$; it is well known that $\varphi=p q \mu+\lambda$. Now $M(\alpha)$ is Seifert fibered with base orbifold $S^{2}(p, q,|\alpha \cdot \varphi|)$ whenever $\alpha \neq \varphi$, according to [Heil 1974]. In the present setting, $\alpha= \pm \mu+n \lambda$ for $n>0$, so $M(\alpha)=S_{ \pm 1 / n}^{3}\left(T_{p, q}\right)$. As a result, $M( \pm \mu+n \lambda)=S_{ \pm 1 / n}^{3}\left(T_{p, q}\right)$ is Seifert fibered with base orbifold $S^{2}(p, q, p q n \mp 1)$ as claimed.

Seifert involutions. For a link $L \hookrightarrow S^{3}$, let $\Sigma\left(S^{3}, L\right)$ denote the two-fold branched cover of $S^{3}$, branched over $L$.

Proposition 2 [Seifert 1933]. $S_{ \pm 1 / n}^{3}\left(T_{2, q}\right) \cong \Sigma\left(S^{3}, T_{q, 2 q n \mp 1}\right)$ for $n>0$ and odd $q>1$.

Proof. The manifold $\Sigma\left(S^{3}, T_{q, 2 q n \mp 1}\right)$ is the Brieskorn sphere $\Sigma(2, q, 2 q n \mp 1)$ and is Seifert fibered with base orbifold $S^{2}(2, q, 2 q n \mp 1)$ [Milnor 1975, Lemma 1.1]; see also [Seifert 1933, Zusatz zu Satz 17]. For each $n>0$ and odd $q>1$, there is a unique $\mathbb{Z}$-homology sphere admitting a Seifert fibered structure with base orbifold $S^{2}(2, q, 2 q n \mp 1)$; see for example [Scott 1983; Saveliev 1999, Theorem 6.7]. The result follows.

The Montesinos trick. A knot $K$ is called strongly invertible if there is an involution of $\left(S^{3}, K\right)$ that reverses orientation on $K$. Thus, the complement $S^{3} \backslash v(K)$ of any strongly invertible knot admits an involution with one-dimensional fixed point set given by a pair of arcs meeting the boundary $\partial\left(S^{3} \backslash v(K)\right)$ transversally in the 4 endpoints. Since the quotient of a solid torus under such an involution is a 3-ball, it follows that a fundamental domain for the action of the involution on $S^{3} \backslash v(K)$ is a 3-ball as well, since $S^{3} \cong \Sigma\left(S^{3}, L\right)$ if and only if $L$ is the trivial knot [Waldhausen 1969].

By keeping track of the fixed point set in the quotient, we obtain a tangle denoted by $T=\left(B^{3}, \tau^{\prime}\right)$, where $\tau^{\prime}$ is a pair of arcs properly embedded in the 3-ball $B^{3}$ meeting the boundary transversally in 4 points. By construction, $S^{3} \backslash v(K)$ is realized as the two-fold branched cover of $B^{3}$, denoted $\Sigma\left(B^{3}, \tau^{\prime}\right)$, branched over the $\operatorname{arcs} \tau^{\prime}$. In this context tangles are considered up to homeomorphism of the pair $\left(B^{3}, \tau^{\prime}\right)$ that generally need not fix the boundary sphere.

Given a strongly invertible knot, the Montesinos trick [1975] amounts to the observation that Dehn surgery in the cover may be interpreted as rational tangle 

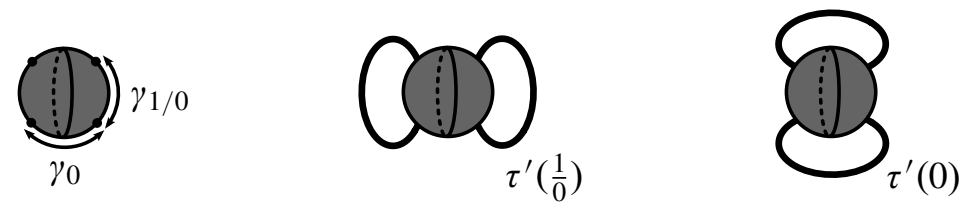

Figure 1. The arcs $\gamma_{1 / 0}$ and $\gamma_{0}$ in the boundary of a tangle (left) lifting to $\mu$ and $m \mu+\lambda$ respectively. The "denominator" (center) and "numerator" (right) closures are denoted by $\tau^{\prime}\left(\frac{1}{0}\right)$ and $\tau^{\prime}(0)$ respectively. Note that in this context $\tau^{\prime}\left(\frac{1}{0}\right)$ is the trivial knot.

attachment in the base. Recall that a tangle is rational if and only if the two-fold branched cover is a solid torus. To identify the corresponding branch set to a given surgery, in Figure 1 we briefly recall the notation introduced in [Watson 2008].

By construction, it is possible to identify the trivial surgery by the unknotted branch set $\tau^{\prime}\left(\frac{1}{0}\right)$ (see Figure 1, and Figure 3 for a particular example). Said another way, the arc $\gamma_{1 / 0}$ in the boundary of this representative for the tangle, identified in Figure 1, lifts to the knot meridian $\mu$ in the cover. Thus, the link $\tau^{\prime}(0)$ gives the branch set for some integer surgery; the arc $\gamma_{0}$ lifts to a slope $m \mu+\lambda$ in $\partial\left(S^{3} \backslash v(K)\right)$ for some $m$, where $\lambda$ is the preferred longitude.

More generally, we may represent any integer surgery by varying the number of half-twists as in Figure 2, since the half-twist lifts to a full Dehn twist about the meridian; see [Rolfsen 1976], for example. As a result it is always possible to fix a preferred representative, which we denote $\left(B^{3}, \tau\right)$, of the homeomorphism class $T$ with the property that $S_{0}^{3}(K) \cong \Sigma\left(S^{3}, \tau(0)\right)$. In this notation, we have that $\tau^{\prime}(0) \simeq \tau(m)$, and the desired homeomorphism is determined by $m$ half-twists. Moreover, $S_{n}^{3}(K) \cong \Sigma\left(S^{3}, \tau(n)\right)$, where $\tau(n)$ is the link shown in Figure 2 .

It is possible to determine the preferred representative directly by carefully keeping track of the image of the preferred longitude in the quotient; see for example [Bleiler 1985]. However, in practice it is straightforward to determine the appropriate homeomorphism after the fact by using the determinant of the link, given that the meridian is easy to identify in this context. Recall that $\operatorname{det} L=$
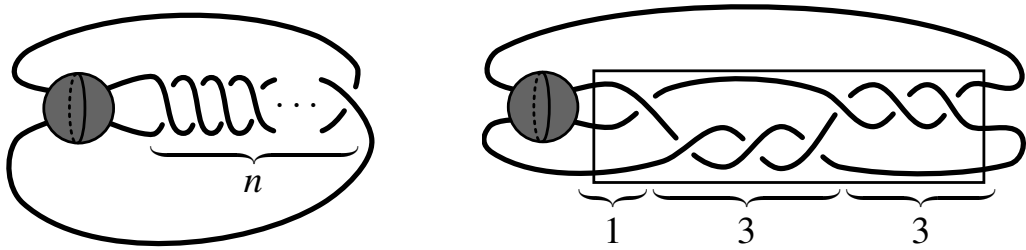

Figure 2. At left, the closure $\tau(n)$ of the preferred representative giving rise to the branch sets for integer surgeries. At right, the closure $\frac{13}{10}=[1,3,3]$ corresponding to $\frac{13}{10}$-surgery in the cover. 
$\left|H_{1}\left(\Sigma\left(S^{3}, L\right) ; \mathbb{Z}\right)\right|$ whenever this group is finite, and $\operatorname{det} L=0$ otherwise. In particular, det $\tau(n)=n$.

More generally, we would like to define the branch set $\tau(r / s)$ for the 3-manifold $S_{r / s}^{3}(K)$, continuing with the notation of [Watson 2008], so that

$$
S_{r / s}^{3}(K) \cong \Sigma\left(S^{3}, \tau(r / s)\right) .
$$

To this end, let $\left[a_{1}, a_{2}, \ldots, a_{m}\right]$ be a continued fraction expansion for $r / s$. Now $\left[a_{1}, a_{2}, \ldots, a_{m}\right]$ encodes a rational tangle that lifts to the desired homeomorphism of the boundary; see for example [Rolfsen 1976]. A specific example is shown in Figure 2. As suggested, the desired homeomorphism is specified by an element of the 3-strand braid group $\left\langle\sigma_{1}, \sigma_{2} \mid \sigma_{1} \sigma_{2} \sigma_{1}=\sigma_{2} \sigma_{1} \sigma_{2}\right\rangle$, where the generator $\sigma_{2}$ lifts to a Dehn twist about $\mu$ and the inverse $\sigma_{1}^{-1}$ lifts to a Dehn twist about $\lambda$. For details on conventions, see [Rolfsen 1976, Chapter 10] and [Watson 2008].

Montesinos involutions. By a result of Schreier [1924], the knot $T_{p, q}$ is strongly invertible. As such, it is possible to realize the manifold $S_{r / s}^{3}\left(T_{p, q}\right)$ as a two-fold branched cover via the Montesinos trick, as outlined above. The goal of this section is to determine the preferred representative of the tangle for which $\Sigma\left(B^{3}, \tau\right) \cong$ $S^{3} \backslash v\left(T_{p, q}\right)$.

In the interest of being explicit, consider the torus knot $K=T_{2,5}$, the knot $5_{1}$ in [Rolfsen 1976]. A strong inversion on this knot is exhibited in Figure 3, together with an illustration of the isotopy of a fundamental domain to obtain a tangle with the property that $S^{3} \backslash v(K) \cong \Sigma\left(B^{3}, \tau^{\prime}\right)$.

We may fix the preferred representative $\left(B^{3}, \tau\right)$ for $T$, as in the previous section, with the properties that

(1) the denominator closure of the tangle, $\tau\left(\frac{1}{0}\right)$, is unknotted and corresponds to a branch set for the trivial surgery, and

(2) the numerator closure, $\tau(0)$, gives a branch set for the zero surgery:

$$
S_{0}^{3}(K) \cong \Sigma\left(S^{3}, \tau(0)\right) .
$$

This representative is shown in Figure 4, and it suffices to verify that det $\tau(0)=0$ (or that det $\tau( \pm 1)=1$ ) to see that this is the preferred representative as claimed. The fact that $\tau^{\prime}(0)$ is a connect sum of 2-bridge links indicates that $\Sigma\left(S^{3}, \tau^{\prime}(0)\right)$ is a connect sum of lens spaces, and hence $\Sigma\left(S^{3}, \tau^{\prime}(0)\right) \cong S_{10}^{3}(K)$. This results from the fact that $\varphi=10 \mu+\lambda$ for the complement of $K=T_{2,5}$ (compare the proof of Proposition 1), and explains the appearance of 10 (negative) half-twists in the preferred representative $\left(B^{3}, \tau\right)$ so that $\tau(10) \simeq \tau^{\prime}(0)$.

See [Montesinos 1976] for a detailed discussion on Seifert fibered spaces as twofold branched covers of $S^{3}$ in general, noting that the Montesinos links shown here encode the Seifert fiber structure in the corresponding two-fold branched cover. 

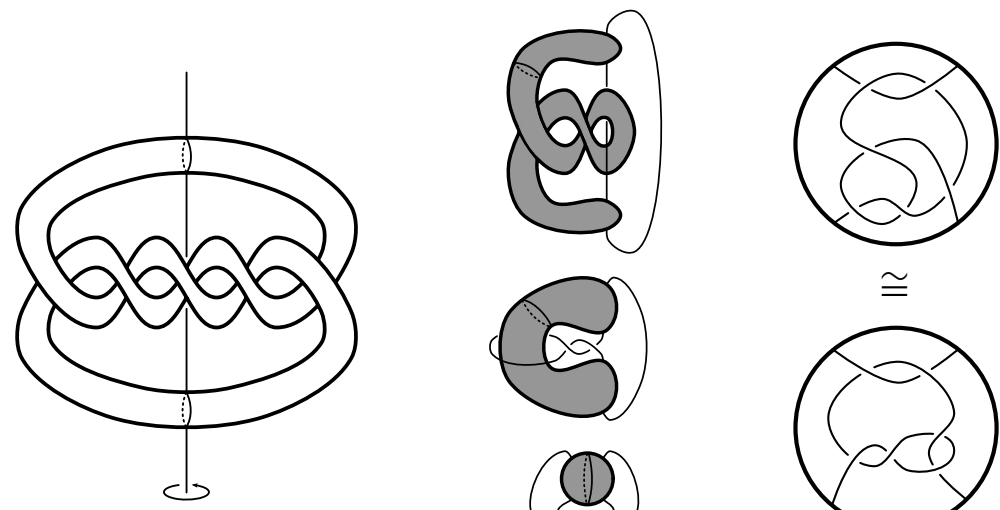

$\cong$
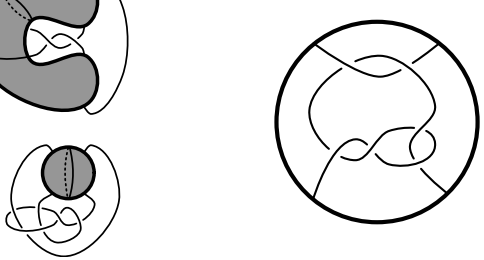

Figure 3. A strong inversion on the torus knot $T_{2,5}$ (left); isotopy of a fundamental domain (center); and two representatives of the associated quotient tangle (right). The Seifert fiber structure on the knot complement is reflected as a sum of rational tangles in the quotient, and the numerator closure in both cases is the trivial knot, identifying the image of the meridian in the quotient.

Proof of the Theorem. Continuing with $K=T_{2,5}$, by the observations above about the Seifert and Montesinos involutions, we have

$$
S_{ \pm 1 / n}^{3}(K) \cong \Sigma\left(S^{3}, T_{5,10 n \mp 1}\right) \cong \Sigma\left(S^{3}, \tau( \pm 1 / n)\right) \text { for } n>0 .
$$

When $n=1$, using the program JavaKh [Bar-Natan and Green 2005], we calculate

$$
\operatorname{rk} \widetilde{\mathrm{Kh}}\left(T_{5,10 \mp 1}\right)=65 \mp 8 \neq 16 \mp 1=\operatorname{rk} \widetilde{\mathrm{Kh}}(\tau( \pm 1)) .
$$

Similarly, when $n=2$ we calculate

$$
\operatorname{rk} \widetilde{\mathrm{Kh}}\left(T_{5,20 \mp 1}\right)=257 \mp 16 \neq 32 \mp 1=\operatorname{rk} \widetilde{\mathrm{Kh}}\left(\tau\left( \pm \frac{1}{2}\right)\right) .
$$

Each of these four pairs of examples illustrates a given manifold as a two-fold branched cover of $S^{3}$ in two different ways, with branch sets distinguished by the total rank of the reduced Khovanov homology. This proves the claim: $\mathrm{rk} \widetilde{\mathrm{Kh}}$ is not an invariant of two-fold branched covers.

Further remarks. We continue with the notation above for the preferred representative of the tangle associated to $T_{2,5}$.

Proposition 3. $\operatorname{rk} \widetilde{\mathrm{Kh}}(\tau( \pm 1 / n)) \leq 16 n \mp 1$ for $n>0$. 

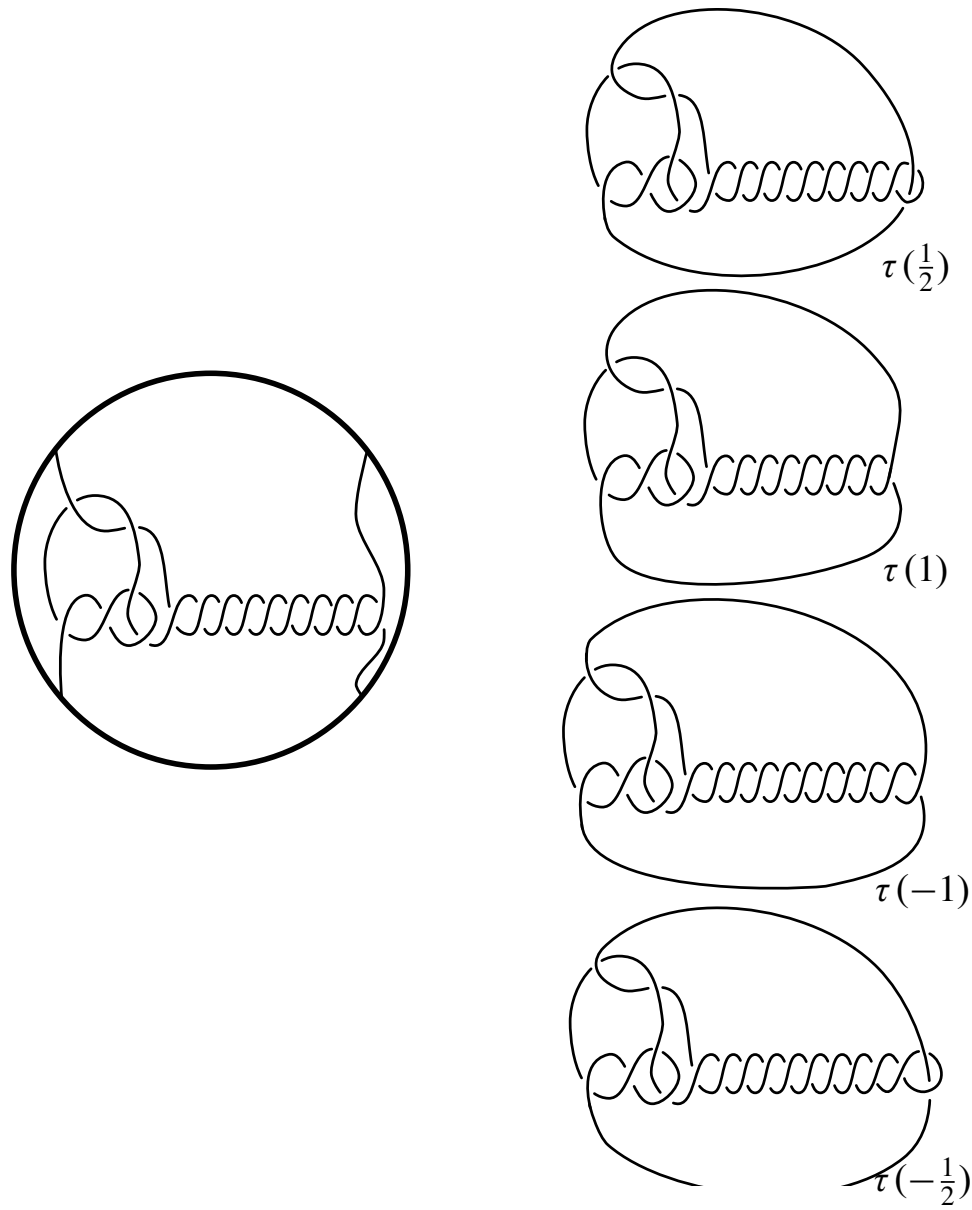

Figure 4. At left, the preferred representative of the associated quotient tangle for the torus knot $T_{2,5}$. At right, the branch sets $\tau\left(-\frac{1}{2}\right), \tau(-1), \tau(1)$ and $\tau\left(\frac{1}{2}\right)$ associated to $\left\{-\frac{1}{2},-1,1, \frac{1}{2}\right\}$ surgery, respectively.

Sketch of proof. We note first that $\mathrm{rk} \widetilde{\mathrm{Kh}}(\tau( \pm 1))=16 \mp 1$, and calculate that $\operatorname{rk} \widetilde{\mathrm{Kh}}(\tau(0))=16$. The result follows by induction on $n$ : Applying the long exact sequence for Khovanov homology, we have

$$
\begin{aligned}
\operatorname{rk} \widetilde{\operatorname{Kh}}(\tau(1 / n) & \leq \operatorname{rk} \widetilde{\operatorname{Kh}}(\tau(1 /(n-1)))+\operatorname{rk} \widetilde{\operatorname{Kh}}(\tau(0)) \\
& =\operatorname{rk} \widetilde{\operatorname{Kh}}(\tau(1 /(n-1)))+16
\end{aligned}
$$

and

$$
\begin{aligned}
\operatorname{rk} \widetilde{\operatorname{Kh}}(\tau(-1 / n)) & \leq \operatorname{rk} \widetilde{\operatorname{Kh}}(\tau(-1 /(n-1)))+\operatorname{rk} \widetilde{\operatorname{Kh}}(\tau(0)) \\
& =\operatorname{rk} \widetilde{\operatorname{Kh}}(\tau(-1 /(n-1)))+16 .
\end{aligned}
$$


On the other hand, calculations of Khovanov homology for large torus knots are difficult to obtain. Indeed, the calculations given here were not accessible prior to the development of JavaKh. However, existing calculations suggest that rk $\widetilde{\mathrm{Kh}}\left(T_{p, q}\right)$ grows at least linearly in $q$. In particular, it seems reasonable to guess that surgery on $T_{2,5}$ provides an infinite family of examples proving the Theorem.

It would be interesting to understand the behaviour of the Khovanov homology for branch sets associated to $(1 / n)$-surgery on the torus knots $T_{2, q}$ for $q \geq 5$.

\section{References}

[Bar-Natan and Green 2005] D. Bar-Natan and J. Green, "Khovanov homology: JavaKh", 2005, Available at http://katlas.math.toronto.edu/wiki/Khovanov_Homology.

[Bleiler 1985] S. A. Bleiler, "Prime tangles and composite knots", pp. 1-13 in Knot theory and manifolds (Vancouver, 1983), edited by D. Rolfsen, Lecture Notes in Math. 1144, Springer, Berlin, 1985. MR 87e:57006 Zbl 0596.57003

[Bloom 2009] J. Bloom, “Odd Khovanov homology is mutation invariant”, preprint, version 2, 2009. arXiv 0903.3746v2

[Boyer 2002] S. Boyer, "Dehn surgery on knots”, pp. 165-218 in Handbook of geometric topology, edited by R. J. Daverman and R. B. Sher, North-Holland, Amsterdam, 2002. MR 2003f:57030 Zbl 1058.57004

[Heil 1974] W. Heil, "Elementary surgery on Seifert fiber spaces", Yokohama Math. J. 22 (1974), 135-139. MR 51 \#11515 Zbl 0297.57006

[Khovanov 2000] M. Khovanov, "A categorification of the Jones polynomial", Duke Math. J. 101:3 (2000), 359-426. MR 2002j:57025 Zbl 0960.57005

[Khovanov 2003] M. Khovanov, "Patterns in knot cohomology, I", Experiment. Math. 12:3 (2003), 365-374. MR 2004m:57022 Zbl 1073.57007

[Milnor 1975] J. Milnor, "On the 3-dimensional Brieskorn manifolds $M(p, q, r)$ ", pp. 175-225 in Knots, groups, and 3-manifolds, edited by L. P. Neuwirth, Ann. of Math. Studies 84, Princeton Univ. Press, 1975. MR 54 \#6169 Zbl 0305.57003

[Montesinos 1975] J. M. Montesinos, "Surgery on links and double branched covers of $S^{3}$ ", pp. 227-259 in Knots, groups, and 3-manifolds, edited by L. P. Neuwirth, Ann. of Math. Studies 84, Princeton Univ. Press, 1975. MR 52 \#1699 Zbl 0325.55004

[Montesinos 1976] J. M. Montesinos, "Revêtements ramifiés de nœuds, espaces fibrés de Seifert et scindements de Heegaard”, lecture notes, Orsay, 1976.

[Moser 1971] L. Moser, "Elementary surgery along a torus knot", Pacific J. Math. 38 (1971), 737745. MR 52 \#4287 Zbl 0202.54701

[Ozsváth and Szabó 2005] P. Ozsváth and Z. Szabó, "On the Heegaard Floer homology of branched double-covers”, Adv. Math. 194:1 (2005), 1-33. MR 2006e:57041 Zbl 1076.57013

[Rolfsen 1976] D. Rolfsen, Knots and links, Mathematics Lecture Series 7, Publish or Perish, Berkeley, CA, 1976. MR 58 \#24236 Zbl 0339.55004

[Saveliev 1999] N. Saveliev, Lectures on the topology of 3-manifolds, de Gruyter, Berlin, 1999. MR 2001h:57024 Zbl 0932.57001

[Schreier 1924] O. Schreier, "Über die Gruppen $A^{a} B^{b}=1$ ”, Hamb. Math. Abh. 3 (1924), 167-169. JFM 50.0070.01 
[Scott 1983] P. Scott, “The geometries of 3-manifolds", Bull. London Math. Soc. 15:5 (1983), 401487. MR 84m:57009 Zbl 0561.57001

[Seifert 1933] H. Seifert, “Topologie Dreidimensionaler Gefaserter Räume”, Acta Math. 60:1 (1933), 147-238. MR 1555366 Zbl 0006.08304

[Waldhausen 1969] F. Waldhausen, “Über Involutionen der 3-Sphäre”, Topology 8 (1969), 81-91. MR 38 \#5209 Zbl 0185.27603

[Watson 2008] L. Watson, "Surgery obstructions from Khovanov homology", preprint, version 3, 2008. arXiv 0807.1341v3

[Wehrli 2007] S. Wehrli, "Mutation invariance of Khovanov homology over $\mathbb{Z}_{2}$ ", lecture notes, Kyoto, 2007.

[Wehrli 2009] S. Wehrli, "Mutation invariance of Khovanov homology over $\mathbb{F}_{2}$ ", preprint, 2009. arXiv 0904.3401

Received September 9, 2009. Revised October 20, 2009.

LIAM WATSON

DEPARTMENT OF MATHEMATICS

UNIVERSITY OF CALIFORNIA

520 Portola Plaza

LOS ANGELES, CA 90095

UNITED STATES

lwatson@math.ucla.edu

http://www.math.ucla.edu/ lwatson 